\title{
Retrieval of wave aberration of human eyes from actual point-spread-function data
}

\author{
Pablo Artal, Javier Santamaría, and Julian Bescós \\ Instituto de Optica, Consejo Superior de Investigaciones Científicas, Serrano 121, Madrid 28006, Spain
}

Received August 31, 1987; accepted April 21, 1988

\begin{abstract}
The wave aberration of human eyes is retrieved from actual point-spread-function (PSF) data and the modulus of the pupil function. The PSF had been obtained previously by application of a hybrid optical-digital method developed recently. The retrieval is done by using a bidimensional Gerchberg-Saxton phase-retrieval algorithm joined to an iterative phase-unwrapping algorithm. To obtain an adequate convergence, the initial wave aberration for starting the retrieval-unwrapping algorithm is estimated with a nonlinear least-squares algorithm. The resulting wave aberrations for several subjects show irregular aberrations superimposed upon the regular waveaberration components, with astigmatism being the most important asymmetric aberration.
\end{abstract}

\section{INTRODUCTION}

Recently a hybrid optical-digital method for the determination of the bidimensional point-spread function (PSF) and the optical transfer function (OTF) of human eyes was presented. ${ }^{1}$ These functions, although they are of great utility for evaluation purposes, do not carry all the optical information, as does the wave aberration.,3

Several workers investigated the determination of the wave aberration of the human eye; Smirnov ${ }^{4}$ measured the wave aberrations of several subjects by using a subjective method and pointed out a typical lack of axial symmetry in the optics of the eye. Howland and Howland ${ }^{5}$ obtained the Taylor polynomial terms to fourth order of the wave aberration with a subjective aberroscope technique.

On the other hand, Berny and Slansky ${ }^{6}$ proposed an experimental method based on the Foucault knife-edge test to determine the wave aberration of the human eye and found important irregular components. Also, Walsh et al. ${ }^{7}$ proposed a method, based on the recording of the retinal image of a grid, that estimates the coefficients of the wave-aberration polynomial through measurements of the grid deformation. In these objective methods, the wave aberration was fitted from indirect measurements, Foucault knife test results, and grid deformations. The availability of direct measurements of the aerial retinal images of a point test led us to retrieve the wave aberration of human eyes from the actual bidimensional PSF, obtained by the above-mentioned hybrid optical-digital method, ${ }^{1}$ and the modulus of the pupil function.

Several methods were proposed for the determination of the wave aberration from PSF data, but the results obtained were successful only with simulated input data. Gonsalves ${ }^{8}$ used the iterative Fourier space-frequency Gerchberg-Saxton (G-S) algorithm ${ }^{9}$ and a parametric search of the Fletcher-Powell-type method. ${ }^{10}$ Maeda and Murata ${ }^{11}$ obtained improved results by using the bidimensional G-S algorithm with simulated data of the PSF but with additional initial information about the type of aberration. Southwell and Nahrstedt ${ }^{12,13}$ proposed a maximum-likelihood algorithm for wave-aberration retrieval from the PSF, and a theoreti- cal study of the nonlinear least-squares-estimation problem for wave aberration was presented in Ref. 14. The retrieval algorithms that were presented previously failed with actual noisy data of the PSF. In this paper we propose a retrieval method that shows good convergence with the actual PSF data and is powerful in any application in the dynamic general problem of wave-front determination when actual PSF data are available.

In what follows, the hybrid optical-digital method for PSF determination is reviewed briefly; the retrieval method for obtaining the wave aberration is described in detail; and wave-aberration results for several subjects, a discussion of the results, and conclusions are presented.

\section{HYBRID OPTICAL-DIGITAL METHOD FOR POINT-SPREAD-FUNCTION DETERMINATION}

The hybrid optical-digital method for the recording of aerial retinal images of a point test was described largely in Ref. 1. Its main characteristics are the use of a $20-\mu \mathrm{m}$ spatial filter pinhole as accommodation and test object, a He-Ne laser beam as the light source, and a calibrated television camera that introduces the short-term aerial images of a point test into a digital image-processing system with a typical exposure time of $1 / 15 \mathrm{sec}$. An averaged aerial image $I\left(x^{\prime \prime}, y^{\prime \prime}\right)$ was obtained by adding a previously estimated number of shortterm images in order to get the image that would be obtained with a long recording time. This is equivalent to considering the second pass an incoherent imaging process. The PSF of the optical system of the eye was then calculated by deconvolution of the resulting averaged intensity $I\left(x^{\prime \prime}, y^{\prime \prime}\right)$. By a subsequent Fourier transformation, the bidimensional OTF was also obtained.

Isometric plots of PSF data obtained by using the procedure outlined above, for three emmetropized subjects in normal sight conditions, are shown in Fig. 1. Subjects PA [Fig. 1(a)] and MA [Fig. 1(b)] were corrected with a -1-D spherical lens and had pupil diameters of $6 \mathrm{~mm}$, and subject JS [Fig. 1(c)] had normal vision and a pupil diameter of 5 $\mathrm{mm}$. 


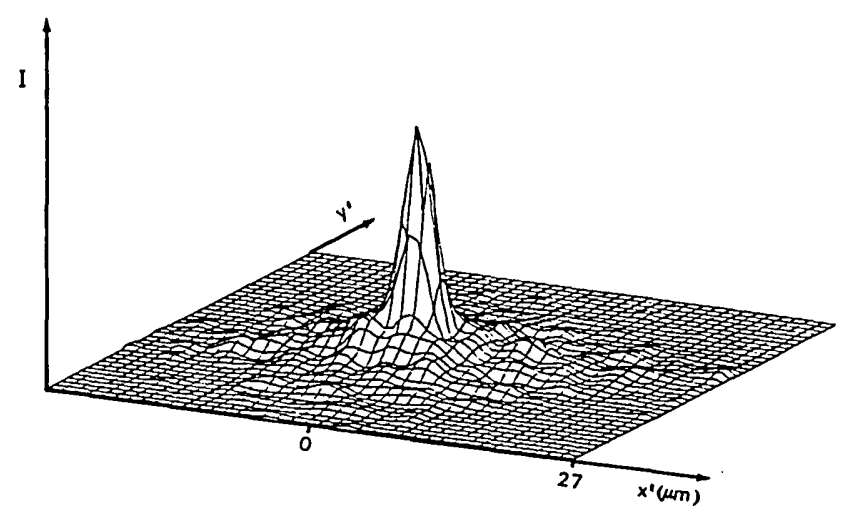

(a)

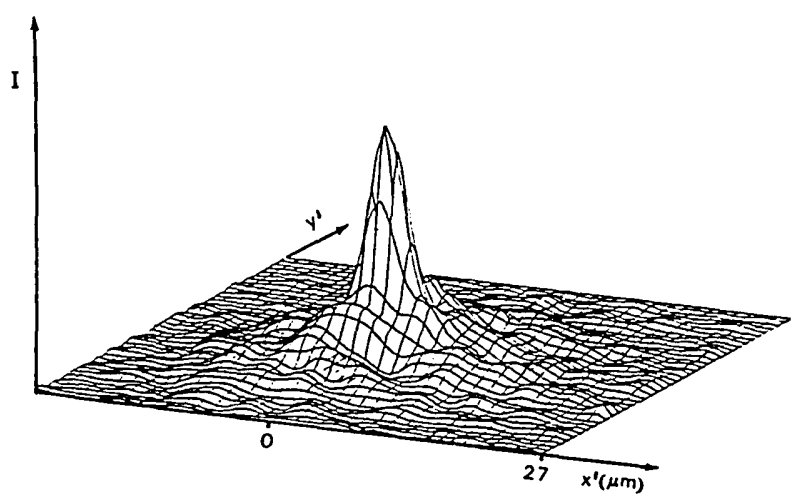

(b)

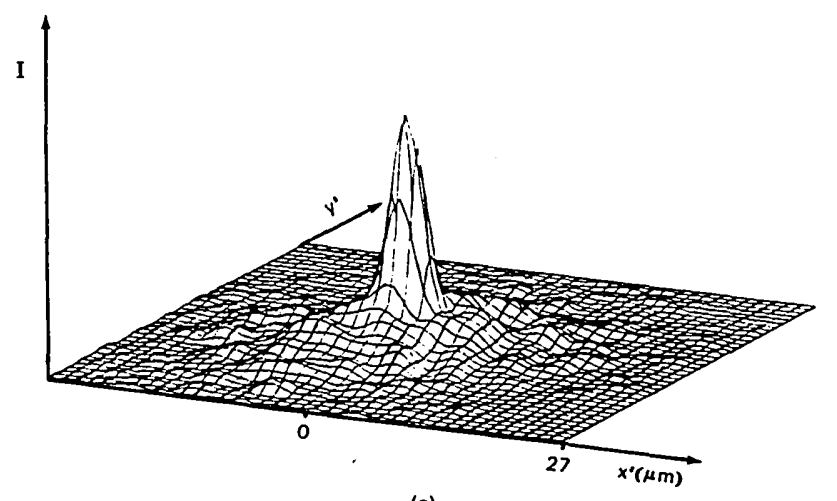

(c)

Fig. 1. Isometric plots of PSF data for subjects (a) PA, (b) MA, and (c) JS.

\section{ALGORITHM FOR RETRIEVAL OF THE WAVE ABERRATION FROM THE POINT-SPREAD FUNCTION}

In order to state the retrieval problem, let us consider the eye optical system, and let $p(\alpha, \beta)$ be the pupil function expressed as

$$
\begin{aligned}
p(\alpha, \beta) & =\exp [(i 2 \pi / \lambda) W(\alpha, \beta)], & & \alpha^{2}+\beta^{2}<1 \\
& =0, & & \alpha^{2}+\beta^{2} \geqslant 1,
\end{aligned}
$$

where $\alpha$ and $\beta$ are normalized pupil coordinates, $\lambda$ is the wavelength of the $\mathrm{He}-\mathrm{Ne}$ laser beam used in the PSF determination, $W(\alpha, \beta)$ is the wave-aberration function, and constant pupil transmission is assumed. The PSF $P_{s}\left(x^{\prime}, y^{\prime}\right)$ is then given by ${ }^{15}$

$$
\begin{aligned}
P_{s}\left(x^{\prime}, y^{\prime}\right)= & \mid \iint_{\alpha^{2}+\beta^{2} \leqslant 1} \exp [(i 2 \pi / \lambda) W(\alpha, \beta)] \\
& \times\left.\exp \left[-i \frac{2 \pi}{\lambda f^{\prime}}\left(\alpha x^{\prime}+\beta y^{\prime}\right)\right] \mathrm{d} \alpha \mathrm{d} \beta\right|^{2},
\end{aligned}
$$

where $x^{\prime}$ and $y^{\prime}$ are retinal-image-plane coordinates and $f^{\prime}$ is the mean focal length of the human eye. ${ }^{16}$

The phase-retrieval problem in this case is to determine the wave-aberration function from PSF data and the modulus of the pupil function $[p(\alpha, \beta)]$. The actual PSF functions were computed previously, and the pupil diameter is also known, so the determination of the wave aberration $W(\alpha, \beta)$ can be solved as a typical double-intensity-measurement phase-retrieval problem.

In this paper the retrieval is done by application of a bidimensional G-S algorithm joined to an iterative phaseunwrapping algorithm ${ }^{17,18}$ because the resulting wave aberration is restricted to values from $-\pi$ to $\pi$. The phaseunwrapping problem arises in homomorphic signal processing ${ }^{19}$ and other technical disciplines and is essentially the determination of the continuous phase curve of a complex function.

The convergence of the algorithm with actual noisy data was poor. To improve it, an initial wave aberration for starting the algorithm was estimated by using an iterative nonlinear least-squares phase-retrieval algorithm, in which a cost function $S\left(a_{i}\right)$ is minimized. This function is defined as

$$
S\left(a_{i}\right)=\frac{\iint\left|P_{s}\left(x^{\prime}, y^{\prime}\right)-P_{s}^{\prime}\left(x^{\prime}, y^{\prime}\right)\right|^{2} \mathrm{~d} x^{\prime} \mathrm{d} y^{\prime}}{\iint\left|P_{s}\left(x^{\prime}, y^{\prime}\right)\right|^{2} \mathrm{~d} x^{\prime} \mathrm{d} y^{\prime}},
$$

where $P_{s}\left(x^{\prime}, y^{\prime}\right)$ is the actual PSF and $P_{s}^{\prime}\left(x^{\prime}, y^{\prime}\right)$ is the PSF corresponding to a wave aberration $W\left(\alpha, \beta, a_{i}\right)$ expressed by an expansion of normalized Zernike circle polynomials ${ }^{2,20}$ :

$$
\begin{aligned}
W\left(\alpha, \beta, a_{i}\right)= & a_{1} \sqrt{5}\left(6 r^{4}-6 r^{2}+1\right)+a_{2} \sqrt{3}\left(2 r^{2}-1\right) \\
& +a_{3} \sqrt{6}\left(\alpha^{2}-\beta^{2}\right)+a_{4} 2 \sqrt{6} \alpha \beta+a_{5} \sqrt{8}\left(\alpha^{2}-3 \beta^{2}\right) \alpha \\
& +a_{6} \sqrt{8}\left(\beta^{2}-3 \alpha^{2}\right) \beta+a_{7} \sqrt{8}\left(3 r^{2}-2\right) \alpha \\
& +a_{8} \sqrt{8}\left(3 r^{2}-2\right) \beta
\end{aligned}
$$

where $r^{2}=\alpha^{2}+\beta^{2}$.

According to previous studies on the performance of the nonlinear least-squares optimization method, ${ }^{21}$ the probability that the algorithm leads to a local minimum of the cost function increases with an increasing number of parameters, so the expansion of the wave aberration in the Zernike circle polynomial is chosen with eight terms as given by Eq. (4).

The convergence in the iterative least-squares method is not good with our noisy input data, but the final results yield an appropriate and independent initial estimation that leads to an improved convergence in the retrieval-unwrapping algorithm. The block diagram of this algorithm is shown in Fig. 2. A complex pupil function is generated by using the known modulus and the initial phase estimation $W_{0}$. This function is Fourier transformed, and the resulting modulus is replaced by the known modulus of the amplitude-spread 


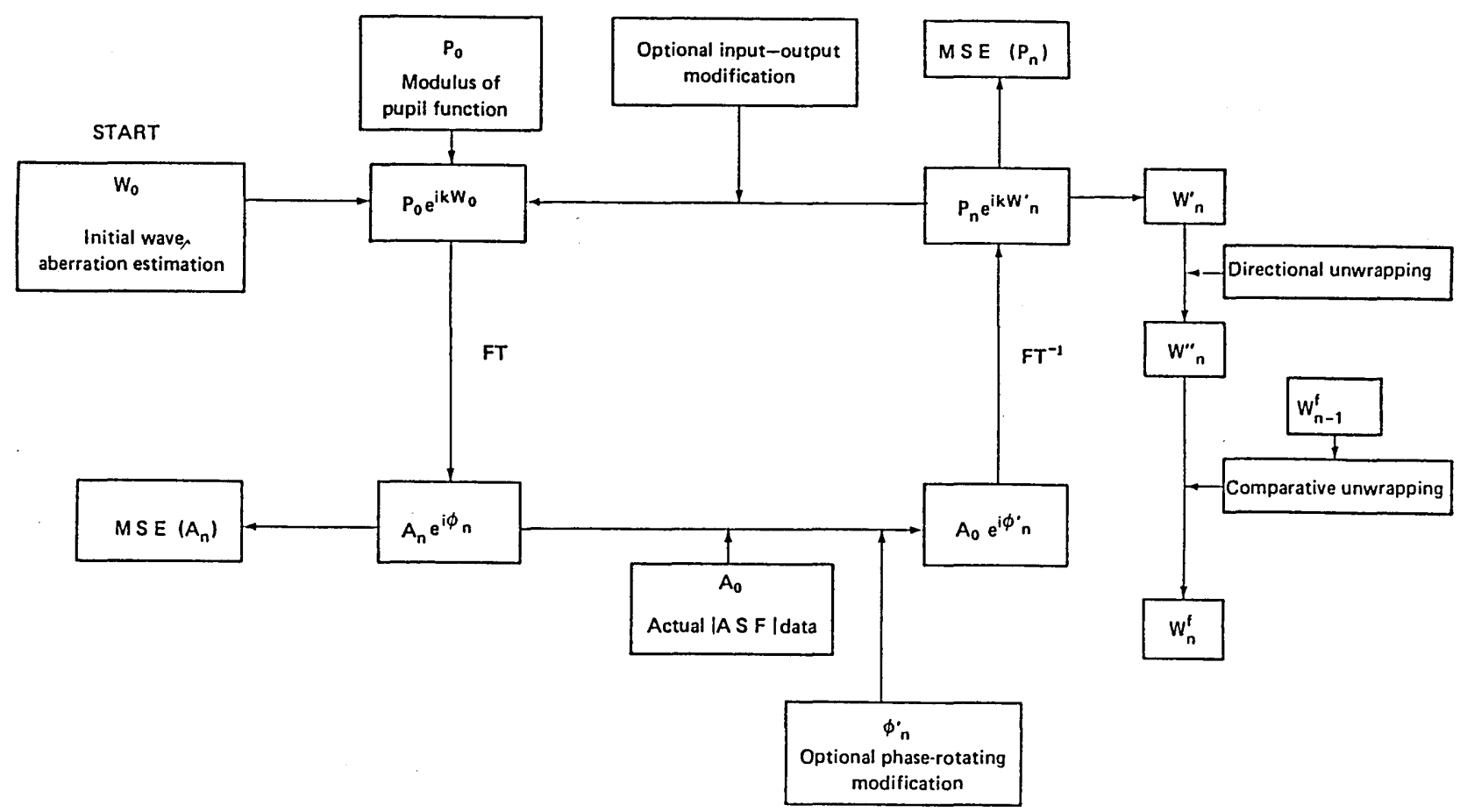

Fig. 2. Block diagram of the iterative wave-aberration-retrieval-unwrapping algorithm. FT, Fourier transform; MSE, mean-square error; ASF, amplitude-spread function.

function $A_{0}$. This complex function is inverse Fourier transformed, and so we obtain a new complex function in which the modulus is replaced by the known modulus $p_{0}$, completing an iteration cycle. Simultaneously, a two-step phase-unwrapping algorithm is performed, consisting of a unidimensional unwrapping by processing of the principal value of the phase (Shafer's algorithm) and then a subsequent comparative unwrapping. In order to avoid typical stagnations, we introduce several modifications to the basic G-S algorithm: an optional phase rotation ${ }^{22}$ and the Fienup input-output modification. ${ }^{23,24}$ However, in our problem improvements of the convergence were not observed, surely because of the noise present in the input data.

In each iteration of the complete algorithm, a meansquared error defined as

$$
\epsilon=\frac{\iint\left|P_{s}\left(x^{\prime}, y^{\prime}\right)-P_{s}^{\prime}\left(x^{\prime}, y^{\prime}\right)\right|^{2} \mathrm{~d} x^{\prime} \mathrm{d} y^{\prime}}{\iint\left|P_{s}\left(x^{\prime}, y^{\prime}\right)\right|^{2} \mathrm{~d} x^{\prime} \mathrm{d} y^{\prime}} .
$$

was computed in the pupil and in the image plane. With about 70 iterations of the algorithm, the mean-square error reached values below 0.05 , even in cases of severely aberrated and noisy PSF data. Arrays with $128 \times 128$ sampling points were used in the computations, and the computing time for the least-squares phase-retrieval algorithm was about 20 min on a Cyber 180/855 computer. Each iteration in the retrieval-unwrapping algorithm took approximately $20 \mathrm{sec}$.

\section{WAVE-ABERRATION RESULTS}

Figure 3 shows the contour plots at $\lambda / 2$ intervals of the resulting wave aberrations retrieved from the actual PSF's corresponding to subjects PA [Fig. 3(a)], MA [Fig. 3(b)], and JS [Fig. 3(c)]. The retrieval was done with 70 cycles in the algorithm, and the final mean-square errors computed in the retinal image plane were $0.057,0.036$, and 0.043 for subjects PA, MA, and JS, respectively. Comparisons between sections of the actual PSF and the resulting PSF obtained with the retrieved wave aberrations by using Eq. (2) are shown in Fig. 4. The plain solid lines correspond to obtained PSF's from retrieved wave-aberration data, and the lines with open circles correspond to the actual PSF.

In order to determine an expansion of the function in Zernike polynomials, yielding information about regular aberrations, a curve fitting on the obtained wave aberration can be done. Table 1 shows the resulting coefficients of Eq. (4) for the three subjects considered in this paper.

If we know the wave aberration of an optical system, we can evaluate the dependence of its image quality on several modifications. This fact is especially important in the case of the human eye for which it is important to be able to predict the change in the image quality when an ophthalmic correction is introduced. In this way, variations of the image quality of human eyes along the visual axis were calculated by adding a continuously variable defocus term to the retrieved wave aberration in order to compute the corresponding PSF where a constant energy level was maintained in the retinal image plane. Figure 5 shows the isometric plot of sections of the bidimensional PSF $\left(y^{\prime}\right)$ distributed along the visual axis $\left(z^{\prime}\right)$ for subject MA.

\section{DISCUSSION}

The hybrid optical-digital method ${ }^{1}$ permits the determination of the retinal image of a point test (PSF). With the availability of these data and with the known pupil diame- 


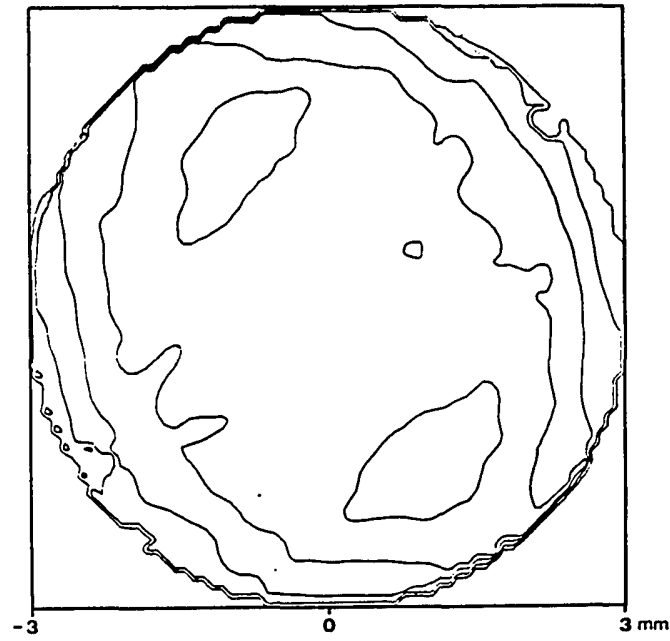

(a)

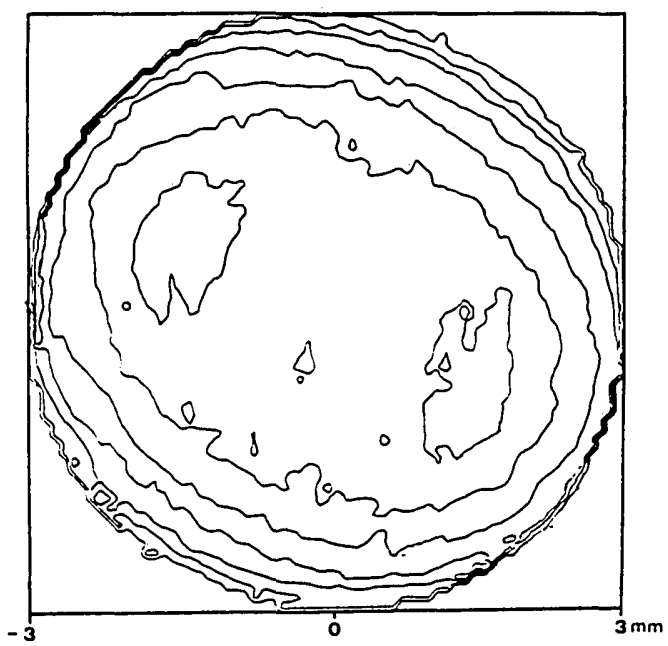

(b)

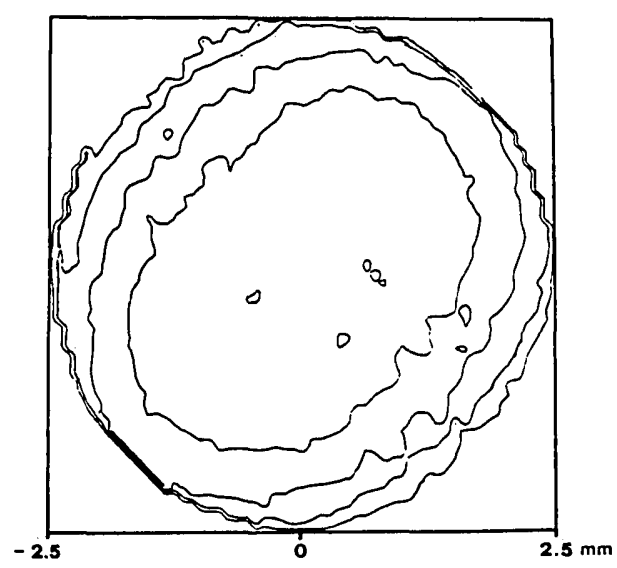

(c)

Fig. 3. Contour plots at $\lambda / 2$ intervals of the retrieved wave aberration for subjects (a) PA, (b) MA, and (c) JS.

ter, the wave aberration for points in the visual axis can be evaluated directly.

The method of wave-aberration retrieval presented here is completely objective, and the quality of the results can also

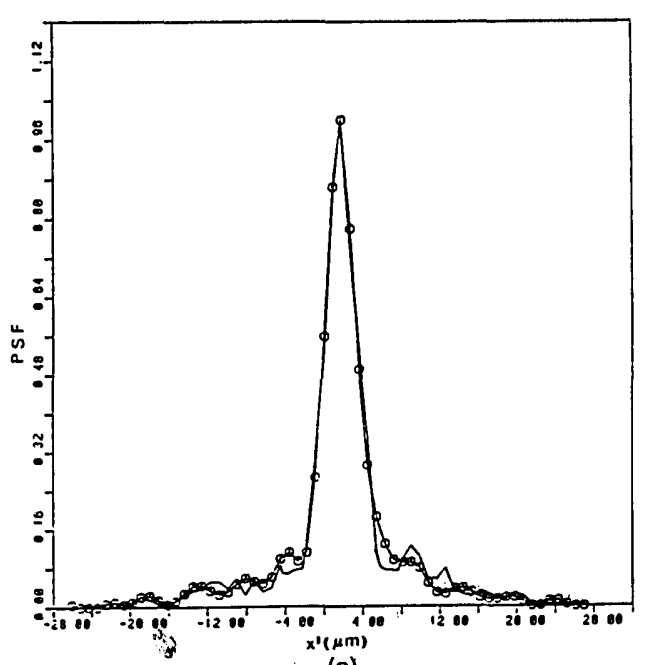

(a)

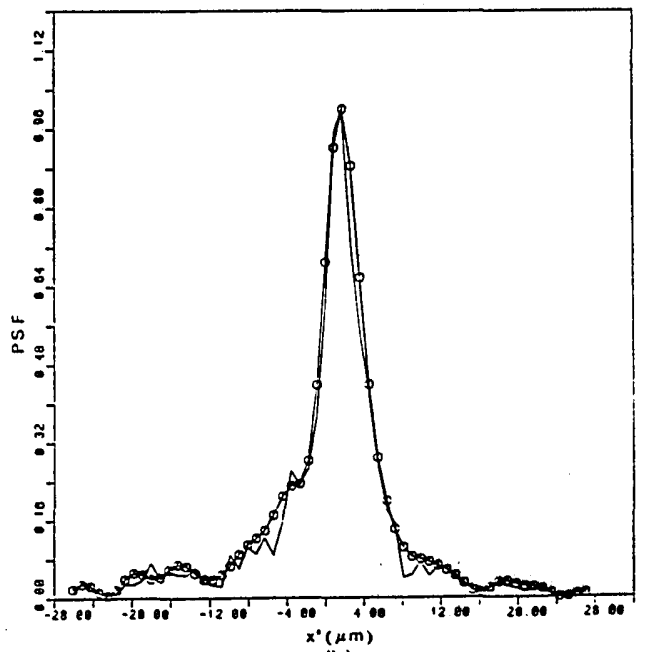

(b)

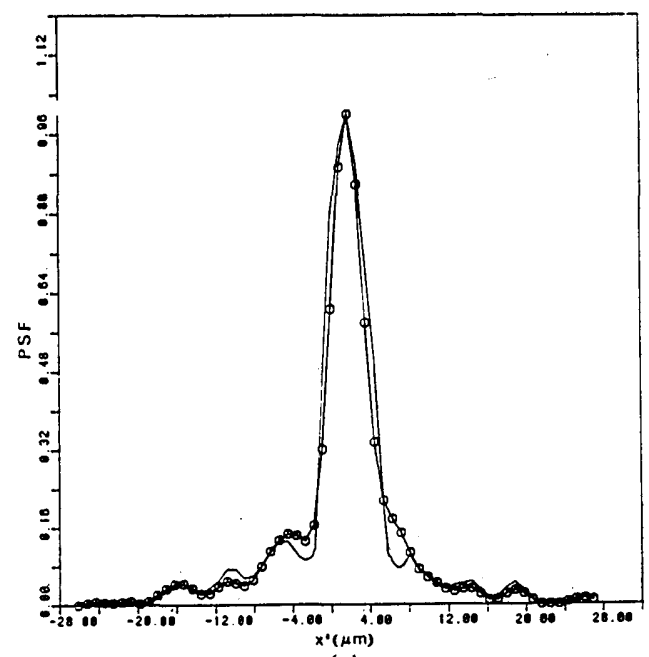

(c)

Fig. 4. Comparisons of a section of the actual PSF (line with open circles) and the resulting PSF (plain solid line) obtained with the retrieved wave aberration for subjects (a) PA, (b) MA, and (c) JS. 
Table 1. Zernike Coefficients of the Regular Aberrations of the Retrieved Wave Aberrations

\begin{tabular}{cccc}
\hline & \multicolumn{3}{c}{ Value for the Following Subject $(\mu \mathrm{m})$} \\
\cline { 2 - 4 } Coefficient & PA & MA & JS \\
\hline$a_{1}$ & 0.099 & 0.076 & 0.089 \\
$a_{2}$ & 0.152 & 0.311 & 0.27 \\
$a_{3}$ & 0.019 & -0.23 & -0.001 \\
$a_{4}$ & 0.134 & 0.18 & -0.001 \\
$a_{5}$ & -0.031 & 0.18 & -0.003 \\
$a_{6}$ & -0.0006 & -0.062 & -0.003 \\
$a_{7}$ & -0.022 & 0.007 & 0.007 \\
$a_{8}$ & -0.008 & 0.0 & 0.0006 \\
\hline
\end{tabular}

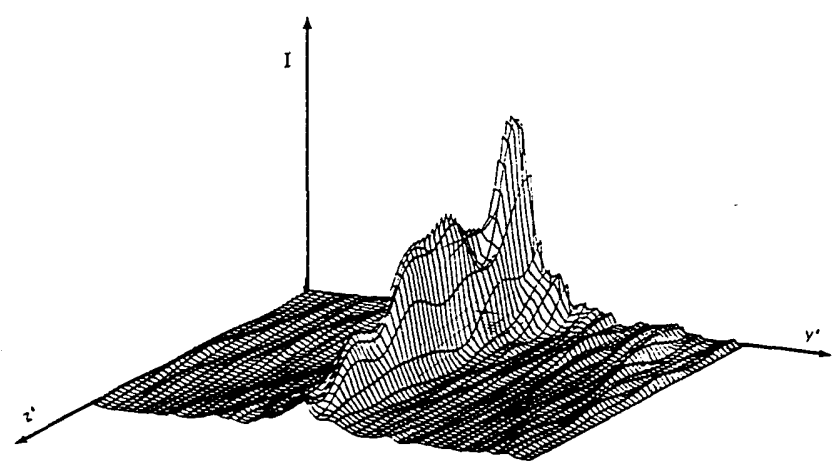

Fig. 5. Isometric plot of the evolution of sections of the PSF $\left(y^{\prime}\right)$ distributed along the visual axis $\left(z^{\prime}\right)$ for subject MA.

be estimated objectively according to an error parameter. This is an important advantage over previous methods for wave-aberration determination ${ }^{4-7}$ in which objective evaluation of the results was not possible.

Irregular aberrations superimposed upon the regular contributions are found in the resulting wave-aberration functions. These irregularities are due to the noise generated by the retrieval method and to the noise present in the input PSF data. In any case, the retrieval-method noise is lower than the value of the mean-square error obtained at the end of the algorithm, and the remaining noise corresponds to irregular aberrations of the eye. Van Meteeren ${ }^{25}$ reviewed the influence of irregular aberrations on the optical quality of the eye and deduced a global effect equivalent to a focal error not larger than $0.15 \mathrm{D}$. Also, previous wave-aberration determinations ${ }^{4,6}$ showed irregularities, but probably an important part would be due to the nonevaluable noise generated by the determination methods.

The regular aberrations were fitted with an expansion of Zernike polynomials [Eq. (4)], a set of orthogonal polynomials over a circular space. The mean-square deviation can then be computed easily from the wave aberration. Several remarks can be made about the results shown in Table 1 . There is considerable dispersion in the results for the individual subjects, even taking into account that all subjects were emmetropized. Several authors ${ }^{4,5,7}$ pointed out the importance of asymmetric aberrations in the optical quality of the human eye. Our results were obtained for points in the visual axis and show a considerable amount of astigmatism, since this aberration is the most important of the asymmetric ones. The comalike components are smaller than the astigmatism, and this is in conflict with the large comalike components found by Howland and Howland 4 and by Walsh et al. ${ }^{7}$ A possible explanation is the influence on these wave-aberration determinations of the off-axis areas of the object grid, depending on the size of the grid used. In any case, our results on the visual axis show small, but nonzero, comalike components, at a level between those of Refs. 5 and 7 and those of Van Meeteren ${ }^{25}$ and Jennings and Charman, ${ }^{26}$ who did not find significant comalike aberrations.

Symmetric aberrations of the eye have been studied largely in the past hundred years. The spherical aberration coefficient obtained in this paper is slightly lower than that obtained previously, ${ }^{16,25}$ surely because asymmetrical aberrations are not usually taken into account, and hence the spherical aberration reaches greater weight.

The phase-retrieval method proposed in this paper can be used successfully with actual PSF data for wave-front-aberration determinations in a number of situations of current interest: for example, image-forming adaptive-optical systems and laser-beam forming. Even with the PSF functions used in this paper, which are severely noisy and aberrated, the convergence of the method is quite acceptable.

\section{CONCLUSIONS}

Wave aberration of human eyes is retrieved successfully from actual PSF data and the modulus of the pupil function. The PSF data had been obtained previously by using a hybrid optical-digital method developed recently. The phase-retrieval method proposed is based on the application of a bidimensional G-S algorithm joined to a phase-unwrapping algorithm. To obtain an improved convergence with our noisy data, the initial wave aberration for starting the algorithm was estimated by using a nonlinear least-squares phase-retrieval algorithm. The retrieved wave-aberration results show considerable dispersion with different emmetropized eyes and irregular aberrations superimposed upon the regular ones. The most important asymmetric regular aberration is astigmatism. This wave-aberration determination method and the hybrid optical-digital method for PSF and OTF determinations permit us to give a more thorough evaluation of the optical image quality for individualized human eyes.

\section{ACKNOWLEDGMENT}

This resarch was supported by the Comision Asesora de Investigación Cientifica y Técnica (grant 2520/83), Ministerio de Educación y Ciencia, Spain.

\section{REFERENCES}

1. J. Santamaría, P. Artal, and J. Bescos, "Determination of the point-spread function of human eyes using a hybrid opticaldigital method," J. Opt. Soc. Am. A 4, 1109-1114 (1987).

2. M. Born and E. Wolf, Principles of Optics (Pergamon, Oxford, 1970).

3. H. H. Hopkins, Wave Theory of Aberrations (Oxford U. Press, London, 1950).

4. M. S. Smirnov, "Measurement of the wave aberration of the human eye," Biophysics 6, 776-794 (1961).

5. H. C. Howland and B. Howland, "A subjective method for the 
measurement of monochromatic aberrations of the eye,"J. Opt. Soc. Am. 67, 1508-1518 (1977).

6. F. Berny and S. Slansky, "Wavefront determination resulting from Foucault test as applied to the human eye and visual instruments," in Optical Instruments and Techniques, J. Home Dickson, ed. (Oriel, London, 1969), pp. 375-385.

7. G. Walsh, W. N. Charman, and H. C. Howland, "Objective technique for the determination of monochromatic aberrations of the human eye," J. Opt. Soc. Am. A 1, 987-992 (1984).

8. R. A. Gonsalves, "Phase retrieval from modulus data," J. Opt. Soc. Am. 66, 961-964 (1976).

9. R. W. Gerchberg and W. O. Saxton, "A practical algorithm for the determination of phase from image and diffraction plane pictures," Optik 35, 237-246 (1972).

10. S. L. S. Jacoby, Iterative Methods for Non-Linear Optimization Problems (Prentice-Hall, Englewood Cliffs, N.J., 1972).

11. J. Maeda and K. Murata, "Retrieval of wave aberration from point spread function or optical transfer function data," Appl. Opt. 20, 274-279 (1981).

12. W. H. Southwell, "Wave-front analyzer using a maximum likelihood algorithm," J. Opt. Soc. Am. 67, 396-399 (1977).

13. D. A. Nahrstedt and W. H. Southwell, "Maximum likelihood phase-retrieval algorithm: applications," Appl. Opt. 23, 43284331 (1984).

14. R. Barakat and G. Newsam, "Numerically stable iterative method for the inversion of wave-front aberrations from measured point-spread-function data," J. Opt. Soc. Am. 70, 1255-1263 (1980).

15. J. W. Goodman, Introduction to Fourier Optics (McGraw-Hill, New York, 1968).
16. R. Navarro, J. Santamaría, and J. Bescós, "Accommodationdependent model of the human eye with aspherics," J. Opt. Soc. Am. A 2, 1273-1281 (1985).

17. D. C. Ghiglia, G. A. Mastin, and L. A. Romero, "Cellular-automata method for phase unwrapping," J. Opt. Soc. Am. A 4, 267280 (1987).

18. J. M. Tribolet, "A new phase unwrapping algorithm," IEEE Trans. Acoust. Speech Signal Process. ASSP-25, 170-177 (1977).

19. A. V. Oppenheim and R. W. Schafer, Digital Signal Processing (Prentice-Hall, Englewood Cliffs, N.J., 1975).

20. S. N. Bezdid'ko, "The use of Zernike polynomials in optics," Sov. J. Opt. Technol. 41, 425-429 (1974).

21. M. Nieto-Vesperinas, "A study of the performance of nonlinear least-square optimization methods in problems of phase retrieval," Opt. Acta 33, 713-722 (1986).

22. R. W. Gerchberg, "The lock problem in the Gerschberg-Saxton algorithm for phase retrieval," Optik 74, 91-93 (1986).

23. J.R. Fienup, "Reconstruction of an object from the modulus of its Fourier transform," Opt. Lett. 3, 27-29 (1978).

24. J. R. Fienup, "Reconstruction of a complex-valued object from the modulus of its Fourier transform using a support constraint," J. Opt. Soc. Am. A 4, 118-123 (1987).

25. A. Van Meeteren, "Calculations on the optical modulation transfer function of the human eye for white light," Opt. Acta 21, 395-412 (1972).

26. J. A. M. Jennings and W. N. Charman, "Off-axis quality in the human eye," Vision Res. 21, 445-455 (1981). 